\title{
Diversity, expression and mRNA targeting abilities of Argonaute-targeting miRNAs among selected vascular plants
}

\author{
Soham Jagtap and Padubidri V Shivaprasad ${ }^{*}$
}

\begin{abstract}
Background: Micro (mi)RNAs are important regulators of plant development. Across plant lineages, Dicer-like 1 (DCL1) proteins process long ds-like structures to produce micro (mi) RNA duplexes in a stepwise manner. These miRNAs are incorporated into Argonaute (AGO) proteins and influence expression of RNAs that have sequence complementarity with miRNAs. Expression levels of AGOs are greatly regulated by plants in order to minimize unwarranted perturbations using miRNAs to target mRNAs coding for AGOs. AGOs may also have high promoter specificity-sometimes expression of AGO can be limited to just a few cells in a plant. Viral pathogens utilize various means to counter antiviral roles of AGOs including hijacking the host encoded miRNAs to target AGOs. Two host encoded miRNAs namely miR168 and miR403 that target AGOs have been described in the model plant Arabidopsis and such a mechanism is thought to be well conserved across plants because AGO sequences are well conserved.

Results: We show that the interaction between AGO mRNAs and miRNAs is species-specific due to the diversity in sequences of two miRNAs that target AGOs, sequence diversity among corresponding target regions in AGO mRNAs and variable expression levels of these miRNAs among vascular plants. We used miRNA sequences from 68 plant species representing 31 plant families for this analysis. Sequences of miR168 and miR403 are not conserved among plant lineages, but surprisingly they differ drastically in their sequence diversity and expression levels even among closely related plants. Variation in miR168 expression among plants correlates well with secondary structures/length of loop sequences of their precursors.

Conclusions: Our data indicates a complex AGO targeting interaction among plant lineages due to miRNA sequence diversity and sequences of miRNA targeting regions among AGO mRNAs, thus leading to the assumption that the perturbations by viruses that use host miRNAs to target antiviral AGOs can only be species-specific. We also show that rapid evolution and likely loss of expression of miR168 isoforms in tobacco is related to the insertion of MITE-like transposons between miRNA and miRNA* sequences, a possible mechanism showing how miRNAs are lost in few plant lineages even though other close relatives have abundantly expressing miRNAs.
\end{abstract}

Keywords: Plant miRNAs, Plant silencing, Argonaute, Dicer-like, miR168, miR403

\section{Background}

Plant miRNAs are indispensable for the control of wide variety of biological functions, including development, hormone responses, feedback mechanisms and biotic and abiotic stresses [1,2]. Most of these functions are associated with ability of miRNAs in targeting mRNAs coding for transcription factors and other key genes [3].

\footnotetext{
* Correspondence: shivaprasad@ncbs.res.in

National Centre for Biological Sciences, GKVK Campus, Bellary Road, Bangalore 560 065, India
}

Nearly half of all known plant miRNAs that are highly conserved across plants target transcription factors [4], justifying their importance in the regulation of plant processes. The remaining half of less-conserved miRNAs regulate expression of a variety of protein coding genes involved in metabolic processes, transporters and the process of RNA silencing itself [4]. Additionally, role of some less-conserved miRNAs in controlling disease resistance in a variety of plants has been recently reported [5-7]. The well-conserved miRNAs that are evolutionarily ancient have many copies in the genomes, 
sometimes up to 50 copies, largely due to genome duplications or due to the rapid evolution of their target mRNAs, or both $[3,4,8]$. The duplicated copies called 'isoforms' may have high similarity in their mature miRNA sequences, but the similarity is less obvious among regions beyond miRNA stem-loops [9-11]. The less conserved miRNAs usually have 1 or 2 copies of themselves with high sequence similarity throughout the length of their RNAs $[3,4,8,12]$.

miRNAs are generated in a stepwise manner from long non-coding, genome-encoded PolII transcripts. Primary miRNA transcripts of varied length must form a secondary structure that can be recognized by Dicer-like protein 1 (DCL1) and its partners. DCL1 complex cleaves the secondary structured RNA to a pre-miRNA structure having a stem with almost complete complementarity and a loop. Another processing step in the cytoplasm produces miRNA:miRNA* duplex of predominantly 21 nts with 2 nt overhangs. While miRNA* is usually degraded, miRNAs associate with Argonaute (AGO) proteins to form RNA-induced silencing complexes (RISC). Most of the plant miRNAs appear to be targeting mRNAs for degradation, typically 'slicing' target mRNA between position 10 and 11. There are also many reports of plant miRNA:mRNA complexes leading to translational inhibition $[1,2]$.

Many factors in miRNA pathway have been characterized, but there is limited information on the selfregulation of miRNA pathway itself. Three miRNAs have been reported to regulate DCL and AGO by cleaving transcripts of corresponding targets as part of a robust feedback mechanism. miR162 has been implicated in targeting DCL1 [13], miR168 in AGO1 mRNA [14-16] and miR403 in targeting AGO2 and AGO3 mRNAs in Arabidopsis and other related plants [17-19]. The regulation of AGOs is quite striking because miRNAs that target mRNAs of AGOs need to form RISC complexes with AGO proteins themselves.

A relatively well-known feedback mechanism involves AGO1 homeostasis that is controlled by coordinated action of miR168 [15,20] and AGO1-derived siRNAs [21] on AGO1 mRNA. Vaucheret et al. [22] also identified an additional complexity of this interaction involving the AGO1-mediated post-transcriptional stabilization of miR168 and the co-regulated expression of AGO1 and miR168 genes in Arabidopsis. Thus, it appears that Arabidopsis has a refined feedback regulatory loop that balances AGO1 and miR168 accumulation. In addition, miR168 expression is regulated by invading viruses. Upon infection with wide range of viruses, miR168 levels go up to dramatic levels quite quickly, leading to the repression of AGO1 translation [16]. A similar upregulation of miR403, though proposed [17], has not been experimentally verified. Regulation of AGOs by miR168 and miR403 has been proposed to be conserved among many plants in addition to tobacco and Arabidopsis, although an in-depth analysis is not forthcoming.

The induction of miRNAs by viruses to meddle with AGO expression indicates that pathogens use these miRNAs particularly to suppress host silencing. This implies that variation in expression levels or targeting abilities of miRNAs among plant lineages has the potential to be the basis of susceptibility or resistance against pathogens. In order to understand these relationships, we analyzed sequence diversity, copy numbers and expression levels of miR168 and miR403 among plant species for which a small RNA dataset is available. Surprisingly, our results suggest that mature sequences of miR168 can be classified into three clades. Presence of three clades is also evident after aligning precursor sequences of these miRNAs. Variation in miR168 sequences seems to correlate well with their AGO1 mRNA targeting abilities. Strikingly, tobacco (N. tabacum) has isoforms with insertion of transposon-like sequences that are likely to reduce their processing, providing a clue to the loss of miRNAs. Furthermore, when we analyzed miR168 sequences across 68 plant species representing almost all major plant families, a clade specific abundance of miR168 was observed. The maximum abundance and diversity of miR168 was among monocots. Monocots have more numbers of AGO1 members than dicots, but few of them are not readily targeted by miR168 creating functional difference. The regulation of AGO2 and AGO3 mRNAs by miR403 is specific to very few plants. miR403 is absent among monocots and many eudicot lineages. Absence of miR403 among monocots indicates an inverse relationship with that of miR168, since miR168 is highly expressed among all monocots studied. In those plants where miR403 is present, the sequences are less diverse and plants have fewer isoforms of this miRNA indicating recent evolution of miR403. Together, this analysis indicates that miR168/ miR403 relationships with their targets as observed in Arabidopsis are likely to be specific only to few plant lineages and plants have evolved every shade of such regulation providing case for altered transcription factor regulations and disease resistance.

\section{Results}

miR168 sequences from diverse plant families fall into three distinct clades

In order to understand the sequence diversity of miR168, we used sequences from miRBase (version 20) as well as from genome-wide transcriptome data reported from plants that have been studied. A total of 58 sequences were obtained representing 31 families of plants. Among these, 16 were newly designated sequences. All 58 miR168 and miR168* sequences were used for sequence alignment (Figure 1, Additional file 1: Table S1) that shows diversity 


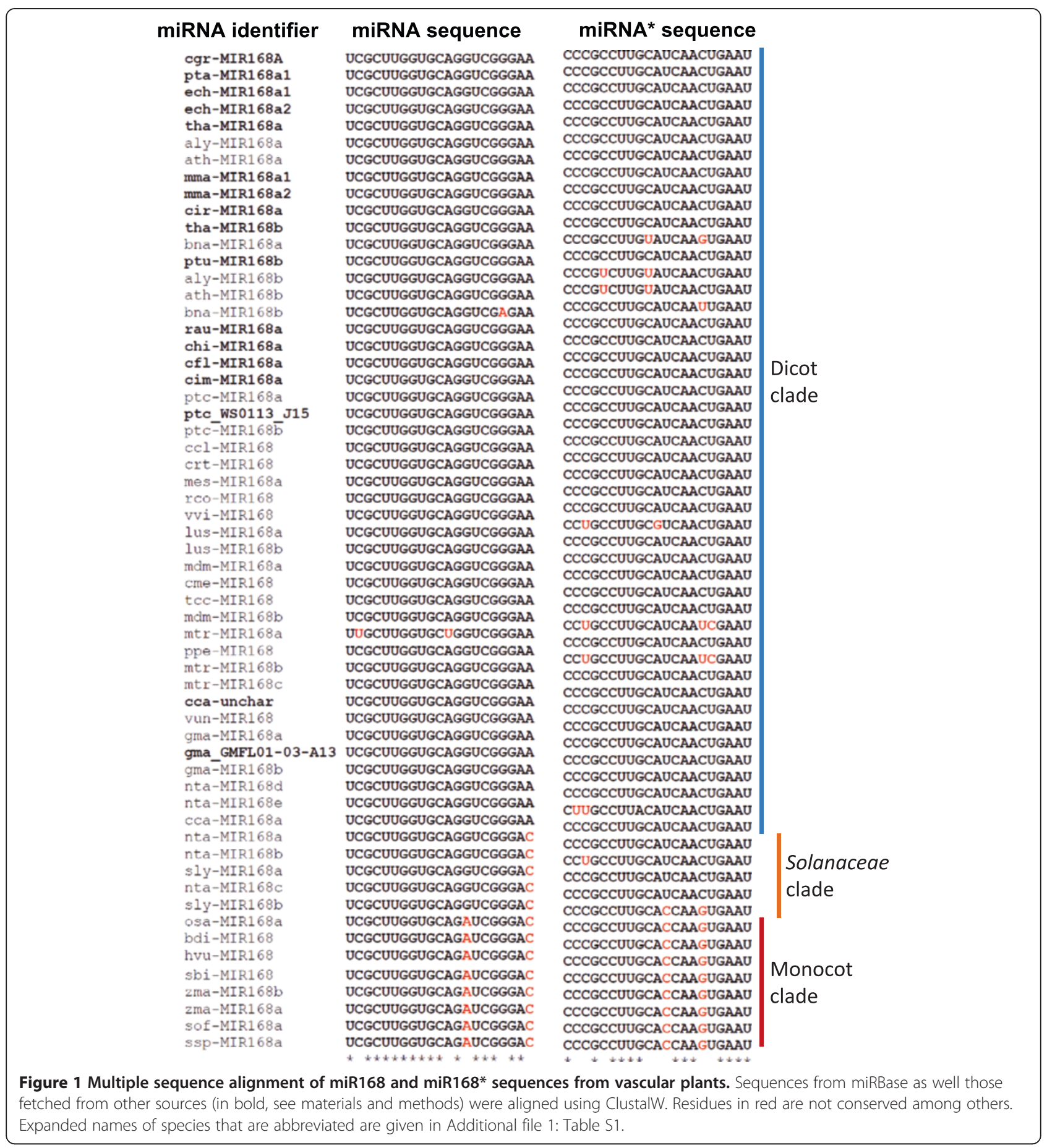

in mature miRNA sequences. Similar miR168 diversity has been documented by a comprehensive analysis reported recently [12]. The mature miR168 sequences can be classified into 3 groups, a large dicot group representing most of the reported miR168 sequences, a monocot-specific group with sequence variations at positions 14 and 21 and a third group of miRNAs with intermediate sequence variation was observed among Solanaceae members. Solanaceae members exhibited similar sequence in $14^{\text {th }}$ position (G) like other dicots, but had similarity at $21^{\text {st }}$ position $(\mathrm{C})$ identical to monocots. The miR168* sequences, on the other hand, had uniform sequence diversity among monocots. Distinct miR168* sequences for Solanaceae members were not observed. Among Solanaceae members, Nicotiana tabacum showed an unusual sequence diversity with two forms (d, e) showing mature miRNA sequences similar 
to other dicots while three additional isoforms (a,b,c) having a Solanaceae specific sequence signature. Two sequences from the dicot clade, miR168 from Brassica napus and Medicago truncatula, had variations that could not be compared to three clades and are likely results of rapid independent evolution.

In order to verify the presence of three groups of miR168 sequences, we aligned the precursors of all miR168 sequences using ClustalW2. Phylogenetic tree derived from this alignment (Additional file 2: Figure S1) also confirms the presence of three clades of miR168 sequences. This suggests that there are sequences beyond mature miRNA sequences that also contribute towards clade-specificity. Presence of these clades and a separate clade for Solanaceae is remarkable because this indicates independent evolution of miR168 among Solanaceae members as these species are distantly related to monocots.

If the miRNAs and their targets co-evolved with their target genes in different plant lineages as proposed [4,23,24], then the target mRNA regions of these miRNAs must have clade-specific changes. However, among the sequences of AGO1 mRNAs from corresponding plant species, there are hardly any clade/family specific changes in the miR168 target regions (Figure 2). The miR168 target region in AGO1 mRNAs does not code for a key RNA motif that will code for a conserved domain, however, there is still high sequence conservation among AGO1 sequences derived from distinct species. This also indicates evolutionarily ancient interaction between miR168 and AGO1. A slightly higher AGO1 sequence divergence in the miRNA target region was observed among phylogenetically unrelated species such as Populus trichocarpa (Salicaceae), Cardamina flexuosa (Brassicaceae), Citrus clementine (Rutaceae), Theobroma cacao (Malvaceae) and Brachypodium distachyon (Poaceae), functional significance of which is unknown.

It is unclear whether miRNAs from one plant species target their mRNAs to the same levels as in other species as such studies have not been carried out. In order to understand the significance of variations in miR168 mature sequences we predicted the miR168 targeting abilities among AGO1 mRNAs of all plant species that have clearly identified miR168 sequences. We used both pssRNAtarget [25] as well tapir [26], two tools that have been acknowledged to provide high reliability [27]. Figure 3 indicates uniformly high targeting (5 or lower tapir score) for most AGO1 mRNAs irrespective of being monocots or dicots, while few showed almost no targeting. Populus trichocarpa, Malus domestica, Theobroma cacao showed very high tapir scores of 10.5, 12 and 7, respectively, and correspondingly low MFe ratios indicating that their AGO1 mRNAs may not be targets of their miR168.

Monocots show higher diversification of AGO1s, usually with 4 members [14], something that may have been due to ancient duplications [28]. We anticipated that presence of additional AGO1 mRNA may provide differential targeting abilities for monocot miR168 members (Additional file 3: Figure S2). Although there are 4 members of AGO1 there are only one or two distinct mature miR168 sequences. Some AGO1 members of Zea mays such as AGO1c and AGO1d are not targeted by zma miR168, but AGO1a and b are good targets (Additional file 3: Figure S2). Rice AGO1d alone has slightly higher tapir score (less optimal target due to mismatch in cleavage site or nearby), while other AGO1 members are good targets. Among the 4 AGO1 members from Brachypodium, one member (Bdi AGO1c) is clearly not a target of bdi miR168 (more than 3 mismatches in seed region). These differences among monocots might contribute to the diversity in their miRNA pathways since AGO1 is a major player in miRNA stabilization and action. We also predicted targets for few miR168 examples from each of the three clades with AGO1 mRNAs from the corresponding plants (Figure $3 \mathrm{C}$ ). This analysis shows that targeting of AGO1 mRNAs by miR168 in monocots is slightly less intense than among dicots based on tapir score.

\section{Secondary structures and precursor miRNA features of miR168s indicate their rapid diversification in Solanaceae clade}

The sequence and distance between miRNAs and miRNA* must indicate evolutionary history of miRNAs [29]. However, these sequences that make up the 'loop' are critical for host DCL1 to process the long non-coding RNAs into short miRNAs duplexes [30]. The distance between miR and $\mathrm{miR}^{*}$ were quite similar among most dicot miR168 precursors, ranging typically between 50 and 80 nts (Additional file 4: Figure S3). Surprisingly, monocots had very short loop sequences in the range of 20-30 (Figure 4A). Among the Solanaceae members there were two distinct groups. One group of precursor sequences that make mature miRNAs common to other dicots, have length of loop sequences between 70-90 typical of other dicots, while some members (nta miR168d and nta miR168e) have unusually long (up to $290 \mathrm{nt}$ ) loops.

Because structures of these miRNA precursors are crucial for their biogenesis, a secondary structure prediction of all miR168 precursors was carried out using RNA-fold (Figure 4B, Additional file 5: Figure S4). Among the dicots, the typical secondary structure had one or two small loops and short branches. Monocots having very short loops had a simple stem loop with high sequence complementarity. Usually high sequence complementarity beyond $\mathrm{miR}$ and $\mathrm{miR}^{*}$ sequences indicate their recent evolution. Among the Solanaceae, those with shorter loops had structures similar to other dicots, but as expected the structures of miR168 isoforms with long loops were complex. The nta miR168d and e precursors have long loop sequences similar to each other but extremely different 

AG01 accession Number
miR168 target site
Tha_AGO1_AM899995.1
122
ACCCCGAGCUGCACCAAGCUA 143
Rau_AGO1_AM899994.1
122 UUCCCGAGCUGCCCCAAGCUA 143
Ptu_AGO1_AM899980.1
122 UUCCCGAGCUGCAUCAAGCUA 143
Mma_AGO1_AM899993.1
122
UUCCCGAGCUGCAUCAAGCUA 143
Ech_AGO1_AM899991.1
128
UUCCCGAGCUGCAUCAAGCUA 149
Cir_AGO1_AM899981.1
125
UUCCCGAGCUGCAUCAAGCUA 146
Cim_AGO1_AM899988.1
122
UUCCCGAGCUGCACCAAGCUA 143
Chi_AGO1_AM899987.1
110
UUCCCGAGCUGCACCAAGCUA 131
Cgr_AGO1_AM899984.1
122
UUCCCGAGCUGCAUCAAGCUA 143
Cfl_AGO1_AM899986.1
56
UUCCGGAACCGCACCAAGCUA 77
CCl_AGO1_XM_006450437.1908
GUCUCUCUCUGCACCAAGCGA 929
Csi_AGO1_XM_006478056.1
677
UUCCCGAGCUACACCAAGCAA 698
Mtr_AGO1_TC126820
305
ACCCCGAGCUGCACCAAGCAA 326
Ptc_AGO1_XM_002329656.1 539
GUGCUGAUCUGCACCAUCUAG 560
TCC_AGO1_XM_007045929.1
3126
Bdi_AGO1C_XM_003573123.1
322
UUUGUAAGGUGCACCAAGCCA 3147
Lus_AGO1_AGO5305
472
CUCCCGACCUGCUGCAGGCUC 343
Ath_AGO1_NM_103737.3
350
UACCCGAGCUGCCCCAAGCUA 493
Aly_AGO1_XM_002894065.1
515
UUCCCGAGCUGCAUCAAGCUA 371
Bna_AGO1_EV190399.1
238
UUCCCGAGCUGCAUCAAGCUA 536
Mdm_AGO1_MDP0000161046 539
UUCCCGAGCUGCAUCAAGCUA 259
Osa_AGO1C_NM_001055156.1 432
UUCCCGACCUGCACCAAGCGA 560
Sbi_AGO1_XM_002437596.1 337
CUCCCGAGCUGCGCCAAGCAA 453
Osa_AGO1D_NM_001065183.1 547
CUCCCGAGCUGCGCCAAGCAA 358
Bdi_AGO1D_XM_003563186.1 316
CUCCCGAGCUGCUCCAAGCAA 568
Bdi_AGO1A_XM_003570001.1407
CUCCCGAACUGCACCAAGCAA 337
Osa_AGO1A_NM_001054239.1 614
UUCCCGAACUGCACCAAGCCC 428
Osa_AGO1B_NM_001187121.1 673
UUCCCGAGCUGCACCAAGCCU 635
Bdi_AGO1B_XM_003573123.1 465
UUCCCGAGCUGCACCAAGCCC 694
Zma_AGO1_AFW75071.2
484
UUCCCGAGCUGCACCAAGCCC 486
Hvu_AGO1_AK373112.1
958
UUCCCGAGCUGCACCAAGCCC 505
Nta_AGO1_AB542739.1
358
UUCCCGAGCUGCACCAAGCAC 979
Sly_AGO1A_NM_001279128.1 533
UACCCGAGCUGCACCAAGCAA 379
UUCCCGAGCUGCACCAAGCAA 554

Figure 2 Alignment of miR168 targeting regions in AGO1 from various plant species. Residues in red are not conserved among others. Start and stop regions in AGO1 mRNAs have been mentioned. 

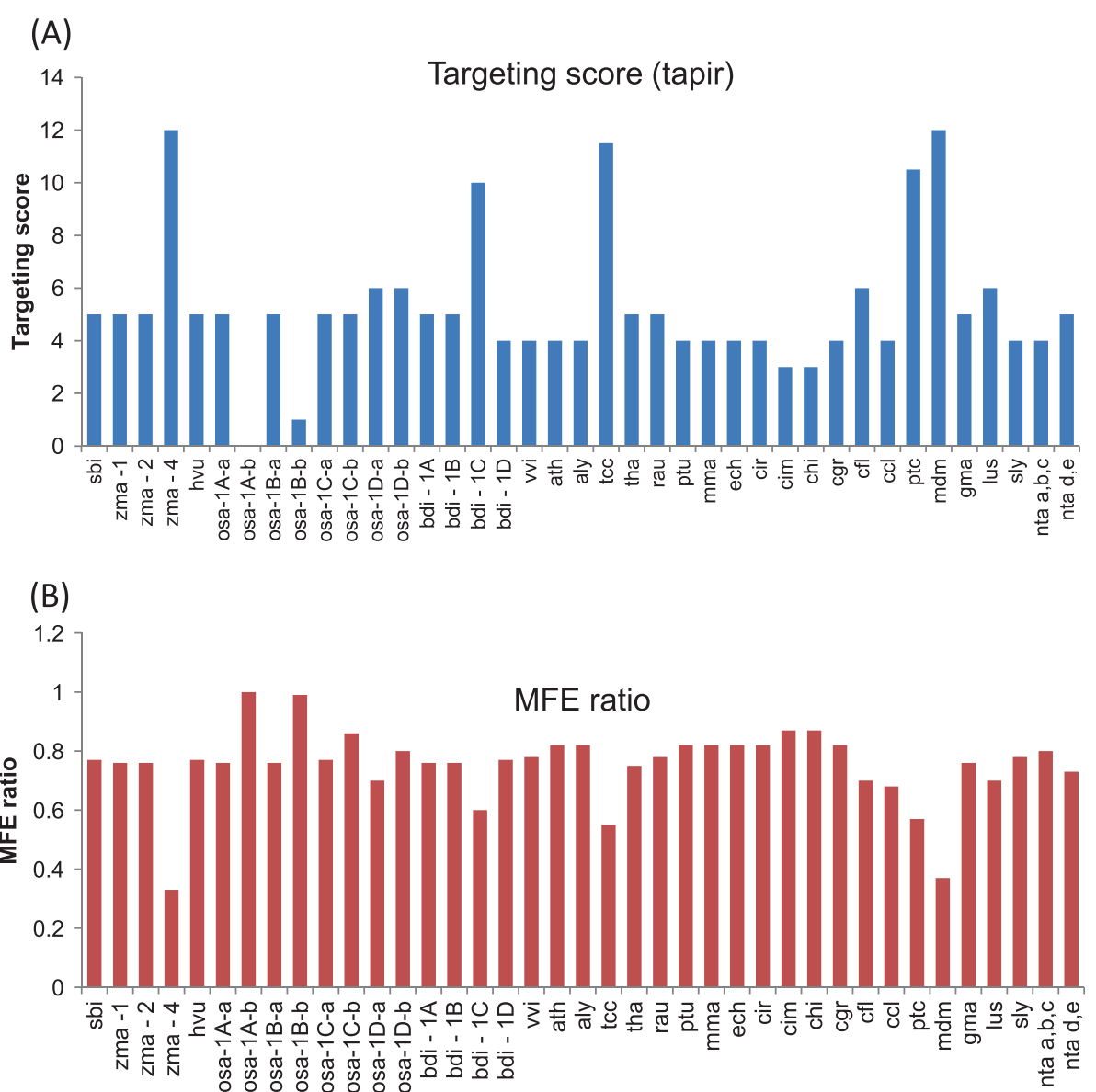

(C)

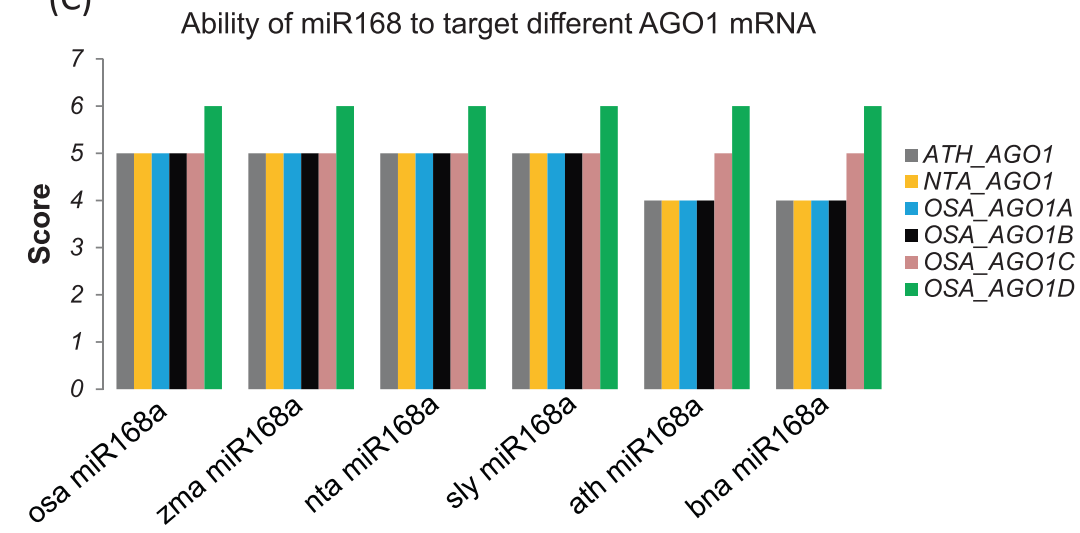

Figure 3 AGO1 targeting abilities of miR168 from corresponding species. (A) Target score for AGO1 targeting in different species by their miR168. TAPIR analysis was carried out as described in methods section. Abbreviation of plant names are given in Additional file 1: Table S1. (B) MFe ratios for the target/miR168 complementarity. Best targets have lower score and higher MFe ratio. (C) Predicted AGO1 targeting of miR168 from few representative species along with AGO1 from other species for comparison.

from any other miR168 precursor (Figure 4C). A closer look showed high sequence similarity between fragments of loop sequences between these two precursors and Miniature Inverted repeat Transposable Elements (MITE) from few dicots (Additional file 6: Figure S5). MITEs are cut and paste type transposon elements typically leaving short fragments when they jump to newer locations. The presence of MITE-like sequences in the loop region for any miRNA has not been reported so far. It is important to note that Piriyapongsa et al. [31] have proposed that miRNAs encoded by MITEs evolved from corresponding ancestral full-length (autonomous) elements that 
(A)

(B)
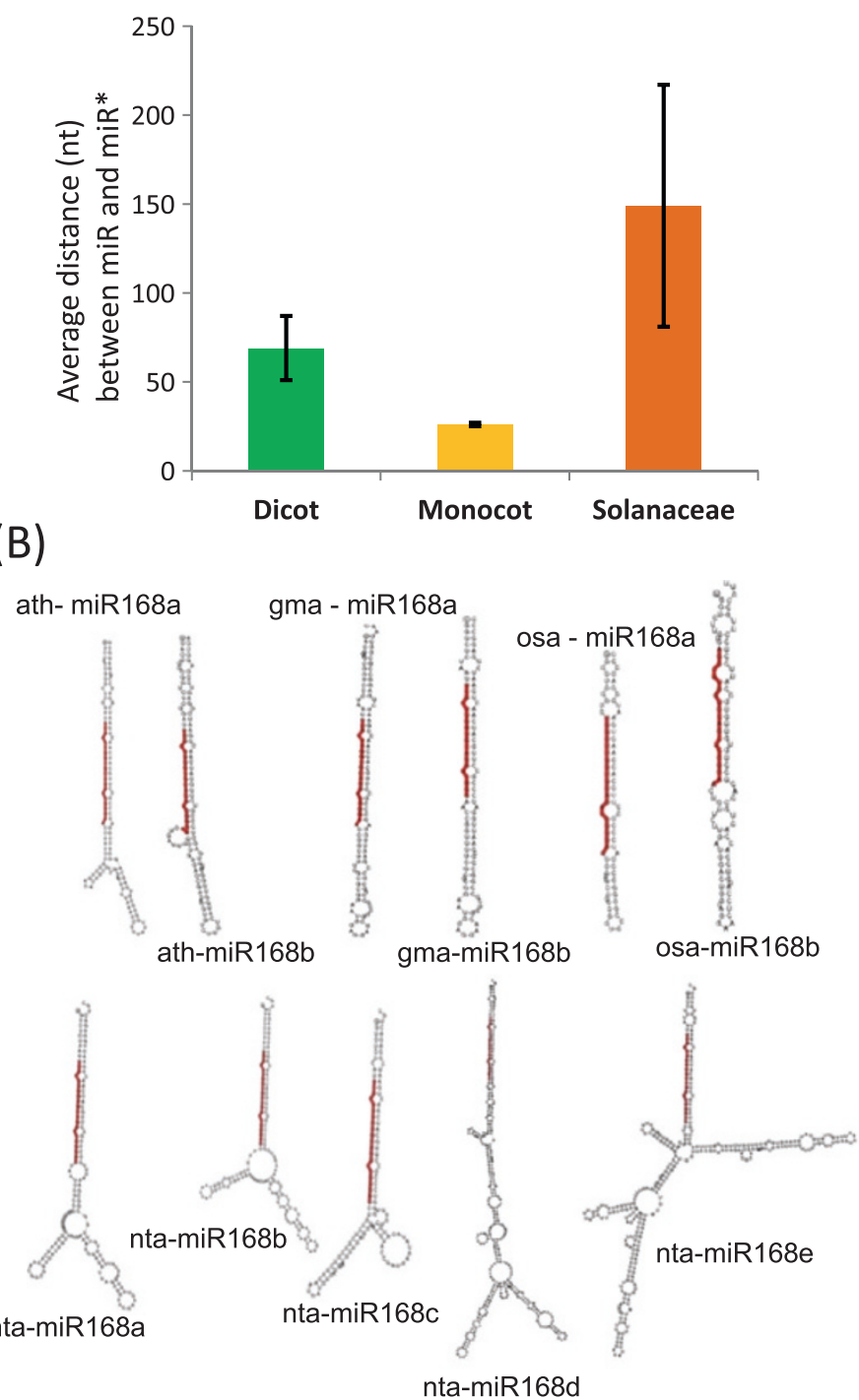

(C)

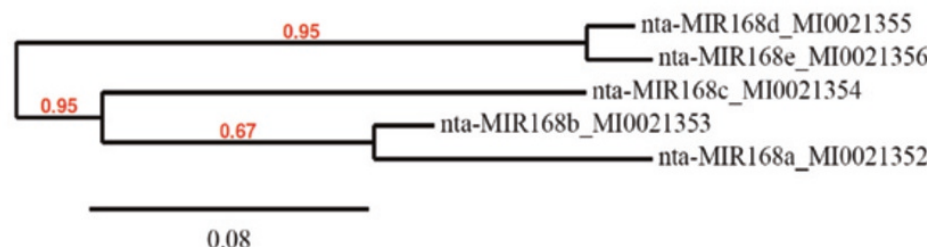

Figure 4 Structural variations in tobacco miR168 isoforms compared to other representative plants. (A) Variation in average length between miR168 and miR168* sequences among monocots, all dicots except Solanaceae and among Solanaceae. (B) Secondary structures of miR168 members from few plants. Although rice, soybean and Arabidopsis have 2 identical mature miR168 isoforms with almost similar secondary structures, tobacco isoforms have diverse secondary structures. RNA fold (http://rna.tbi.univie.ac.at/cgi-bin/RNAfold.cgi) was used to determine secondary structures. (C) Phylogenetic analysis of miR168 isoforms from tobacco indicating two clusters. Tree was constructed as described in methods section.

originally encoded short interfering (si)RNAs. For miR168 though this may have been in a reverse order. A systematic search using published genomes identified other regions that could have been miR168 precursors that invited other repeat elements to become transcriptionally inactive (data not shown). 
In tobacco, identification of mature miRNAs corresponding to isoforms a, b, c (without MITE insertion) and d, e (with MITE insertion) is possible due to the sequence divergence between these isoforms. We hypothesized that insertion of MITEs might interfere with Pri-miR168 transcription or biogenesis steps and therefore those isoforms with insertion should be less abundant compared to their counterparts. Strikingly, tobacco miR168d,e isoforms were $\sim 15$ times low abundant than $\mathrm{a}, \mathrm{b}, \mathrm{c}$ isoforms in leaves and flowers (Figure 5), supporting the idea that long loop-containing precursors of miR168 yield less abundant mature miRNAs.

\section{Comparative abundance of miR168 among plant families}

We used small RNA datasets derived from 42 plant species representing 25 plant families to compare expression and diversity of miR168 sequences. Next generation small RNA sequence datasets from the corresponding species were taken from GEO and other published sources (See materials and methods). The abundance of miR168 and those reads that match to miR168 with 1 or 2 mismatches were taken into consideration ('miRProf' tool, [32]) as genomes of many of these plants and information about their miRNAs are not readily available. The data presented in Figure 6A indicates that there is very high expression of miR168 sequences among most monocot families when compared to dicots. The most abundant form of miR168 among Poaceae was the monocot-specific form of miR168 (Figure 1, Figure 6B). Similar observations were made in a recent study that

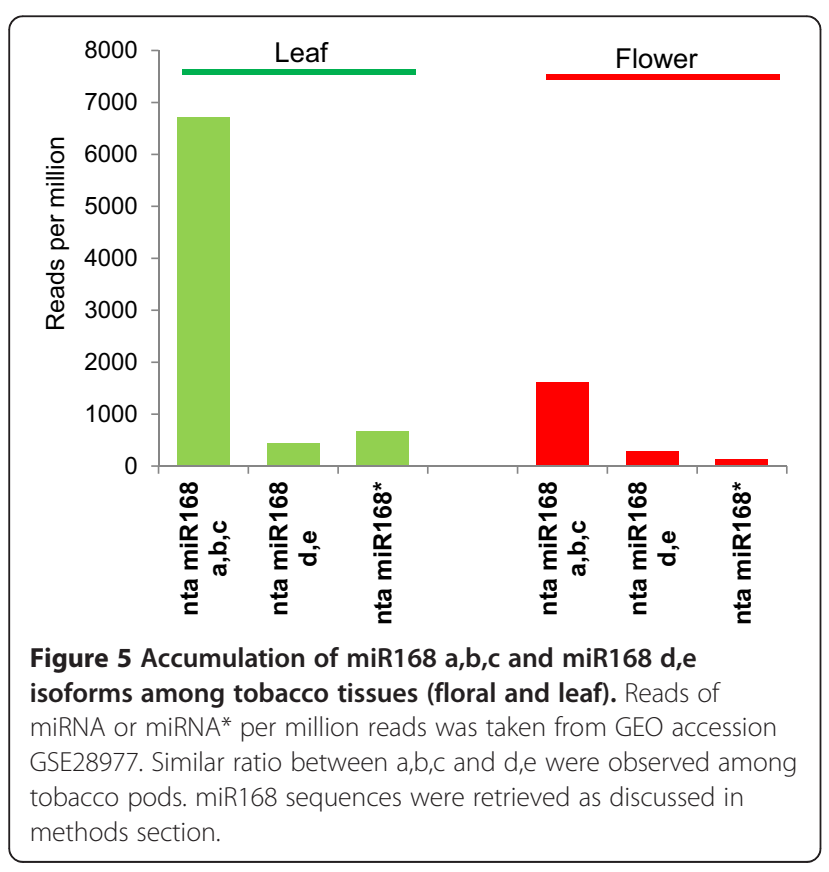

compared miRNA diversity across vascular plants [12]. The other major monocot order Zingiberales (Musa acuminata) on the other hand, seem to have the common dicot specific form as the most abundant. Musa not only has a dicot specific form as most abundant form, but also has accumulation of miR168 levels matching those of dicots in that it has low accumulation. The monocot specific form surprisingly is found also among Cycads (Cycas rumphii), Gingkophyta (Gingko biloba) and Pinophyta (Picea abies). These species represent forms that are ancient to monocot/dicot divergence and it is easy to speculate that these two forms are ancient. Magnoliids (Aristolochia and Persea) show abundance of either mature miR168 form depending on the species. Depending on the plant lineage some miR168 forms could have evolved and expressed better than the other forms. Vitis vinifera (Vitales), a eudicot, has higher expression of monocot specific form unlike other eudicots for those a sequence information is available, is an example wherein both forms co-exist.

Surprisingly, abundance of miR168 was comparatively low among Arabidopsis and members of Solanaceae such as Solanum spp. and Nicotiana spp. Most abundant form of miR168 among Solanaceae was the form that is distinct from other dicots. These forms have also been reported recently in a global study using small RNA datasets from many vascular plants [12]. Among dicots, some Astrids such as Mimulus guttatus (Lamiaceae), Lactuca sativa (Asterales) have the highest accumulation of miR168.

Consistent with the observation in Arabidopsis that floral tissues accumulate more 24 nt siRNAs than miRNAs, miR168 levels are generally low among floral samples of all plant species analyzed. Unexpectedly, rice (Oryza sativa) and sorghum (Sorghum bicolor) are some of the few species where miR168 was consistently expressed highly among both leaf and floral tissues (Additional file 7: Figure S6).

Viruses as part of their counter-defense strategy target the host AGOs by destabilizing them directly at the protein level [33-36]. Some viruses even act at a higher level by inducing degradation of mRNAs of AGO family members that are part of the host defense, namely AGO1, AGO2 and AGO3. Viral counter-defense by targeting of AGO mRNAs is through induction of miR168 as observed in N. benthamiana [16], A. thaliana [22] and S. lycopersicum [16]. After analyzing the global miR168 levels in these species it makes sense to hypothesize why viruses need to upregulate miR168 to target AGO1 mRNAs. All these species have very low levels of miR168 in uninfected tissues indicating they may have higher accumulation of AGO1 protein thereby indicating AGO1 as the most likely and potent candidate to target viruses. However, such an interaction seems unlikely for monocots (these plants act 

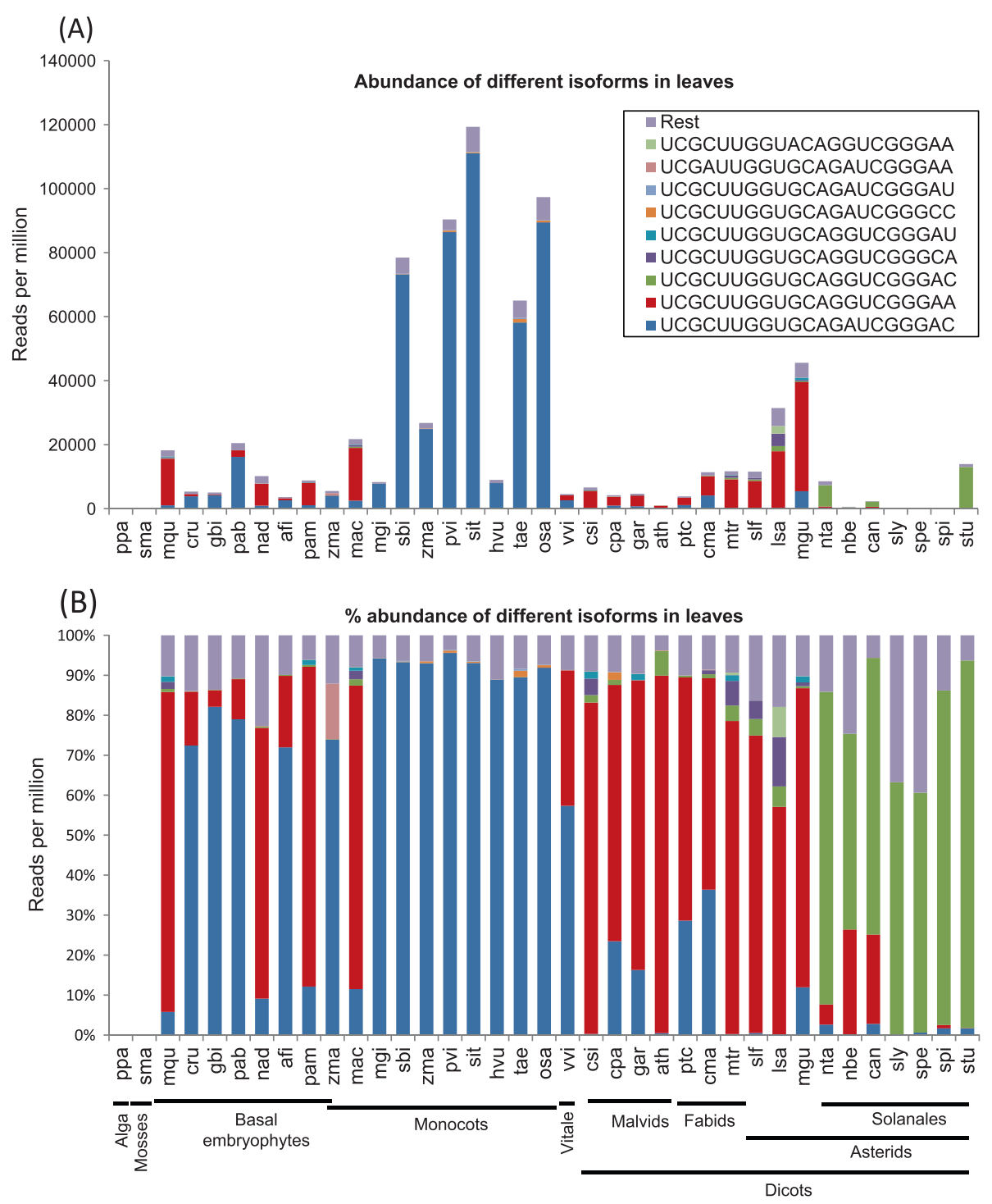

Figure 6 Abundance and sequence diversity of miR168 members across plant families. (A) Highest abundance of miR168 among monocots. Color bars represent most abundant forms of miR168 in leaf tissues. Blue, red and green bars represent monocot, dicot and Solanaceae-specific forms as shown in Figure 1. Abundance was measured as discussed in methods section. (B) Percentage abundance of miR168 across plant families. Phylogenetic relationships among plant species have been indicated.

as hosts to few viruses), members of which accumulate miR168 at higher levels even without any biotic stress. By analyzing publicly available small RNA datasets we can show that miR168 induction upon virus infection of rice (a monocot) is hardly noteworthy ([37] Additional file 8: Figure S7). Corresponding levels of increase in miR168 levels upon viral infection for few dicots such as $N$. benthamiana, Arabidopsis and tomato are at much higher levels [16]. This difference in miR168 levels upon virus infection among dicots and monocots is important because AGO1 protein levels go down dramatically upon virus infection in Arabidopsis and Nicotiana members [16], while in rice, the reduction in AGO1 protein level is negligible
[38]. These results suggest a functional difference in downstream activities of AGO1 among monocots and dicots that is brought about by variation in expression levels of miR168.

miR403 has low sequence diversity and are present only among selected lineages of plants

A similar search for miR403 in miRBase as well as transcript databases recovered 35 sequences (Figure 7). miR403 has been reported to be absent in monocots $[4,39]$, but a detailed information of plant families where this miRNA is present is not known. Among the plants where miR403 is reported, Glycine max alone has a 


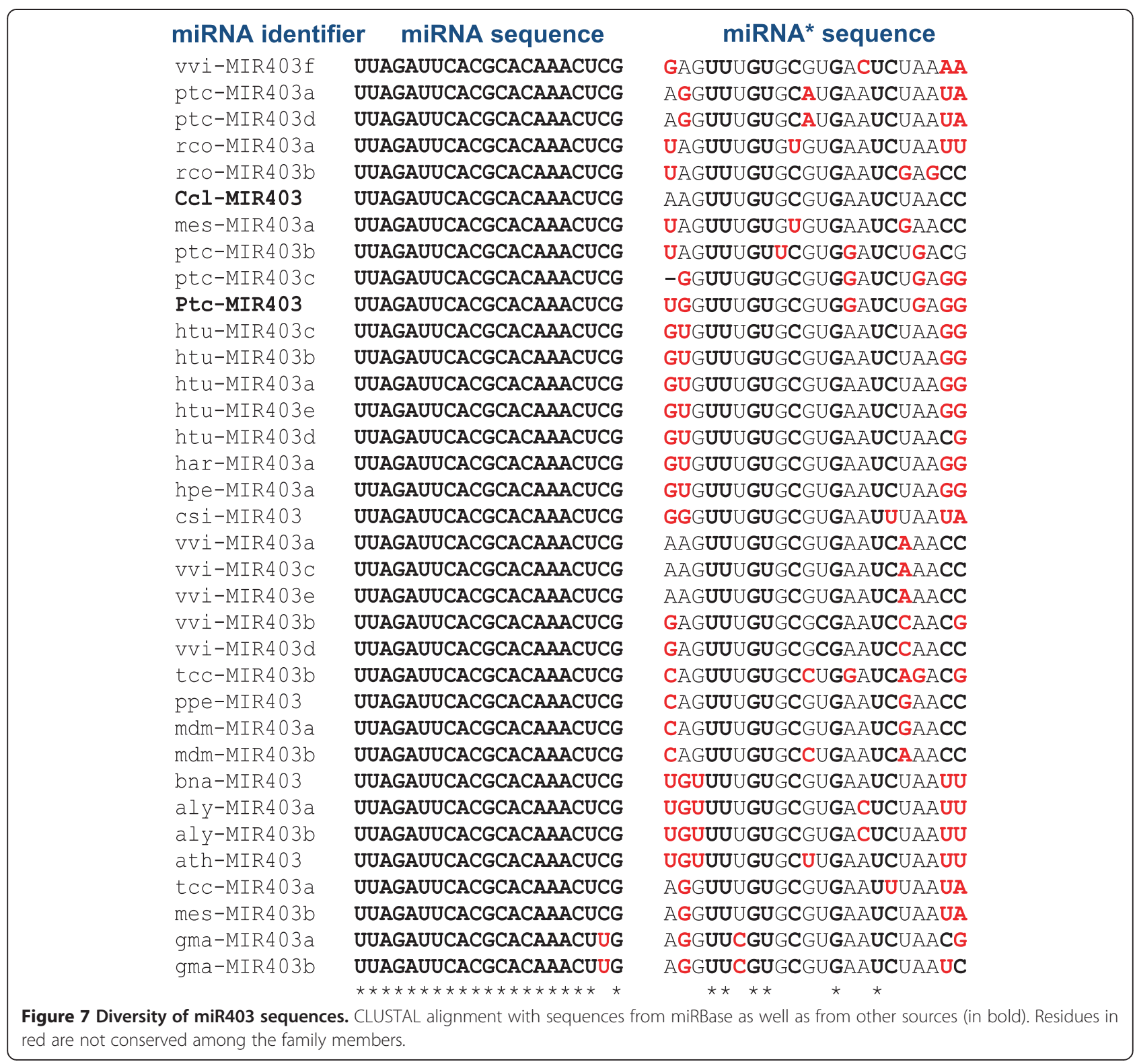

sequence variation (at position 20) when compared to all other species. This lack of diversity in sequences arises from its recent evolution as this miRNA is present only in few lineages of eudicots. Some plant species have many copies of miR403, for example Vitis has 5 copies, all with the same mature sequence but with diverse $\mathrm{miR}^{*}$ sequences. The most common and abundant form of the miR403 is 5'-UUAGAUUCACGCACAAACUCG-3'. Few interesting deviations from this sequence were observed in Solanaceae members (S. pennellii and $S$. tubersum) and some Malvids (Carica papaya) sharing 5'-CUAGAUUCACGCACAAACUCG-3' as the major miR403 isoform. This isoform is extremely interesting in that it has an unusual 5' terminal nucleotide as C. One more abundant form specific to Gossypium arboreum is 5'-UUAGAUUCACGCACAAACUCA-3', thus, Malvids seem to have the most diversity in miR403 sequences.

Surprisingly, while two sets of Rosiids called Malvids and Vitales have abundant miR403, some Fabids (Cucurbita, Phaseolus and Medicago spp.) have no expression of miR403 (Figure 8). However, Populus trichocarpa (Salicaceae), a member of Malphigiales that belong to Fabidae have high expression of miR403. Vitales have very high expression both in vegetative and reproductive tissues (Additional file 9: Figure S8), followed by few Solanaceae members (Solanum and Nicotiana spp.). 


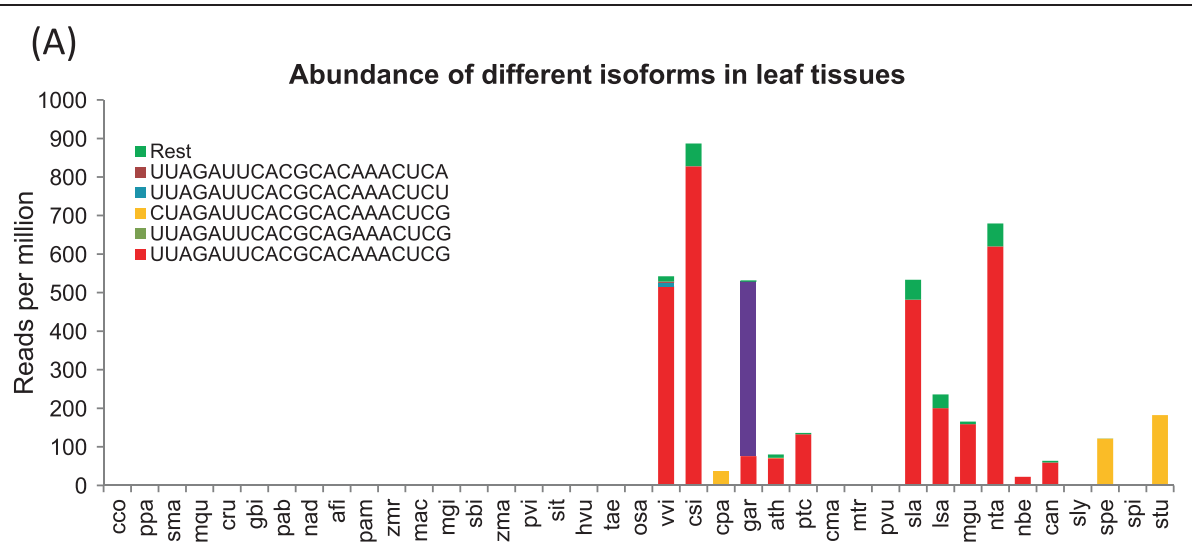

(B)

$\%$ abundance of different isoforms in leaf tissues

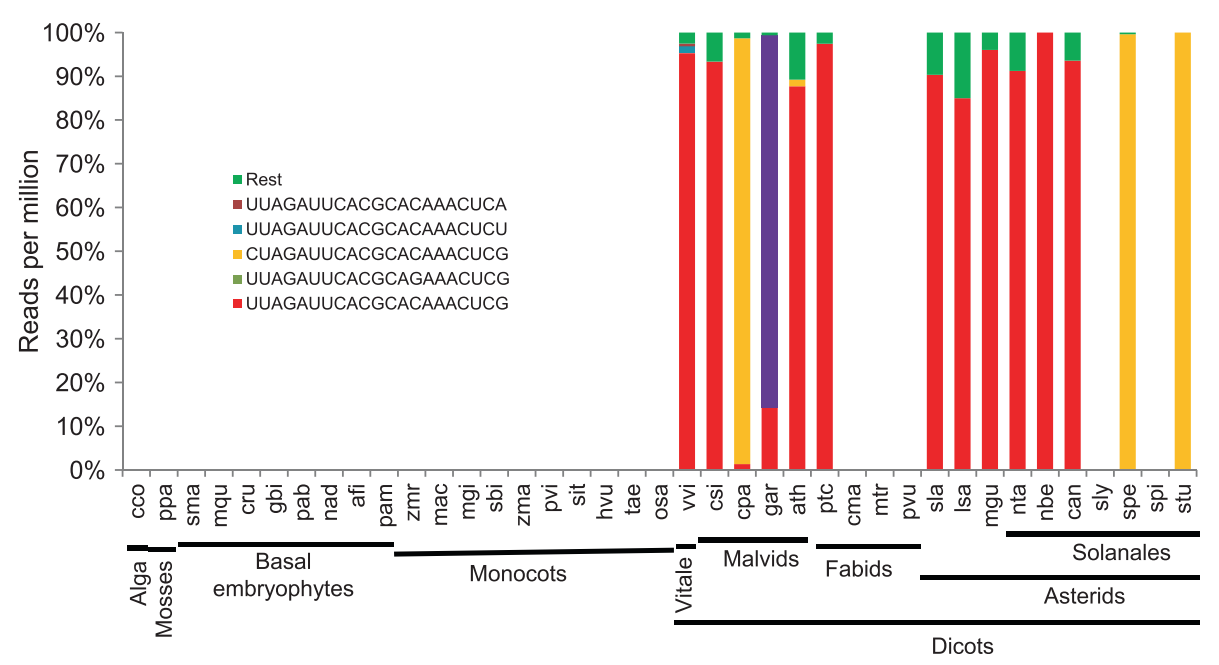

Figure 8 Abundance and sequence diversity of miR403 members across plant families. (A) miR403 is conserved only among Rosiid members namely Malvids and Vitales and few Fabids. Color bars represent most abundant forms of miR403 in leaf tissues. Abundance was measured as discussed in methods section. (B) Percentage abundance of miR403 across plant families.

Targeting of AGO2 and AGO3 mRNAs by miR403 is restricted to few plant lineages

miR403 targets mRNAs of AGO2 and AGO3 in Arabidopsis. It has been proposed that viruses may interfere with miR403 expression in order to reduce the expression of AGO2, considered as a major antiviral AGO. When we analyzed AGO2 mRNAs from those species where miR403 accumulation could be detected, it was observed that targeting of AGO2 mRNA is very efficient in Brassicaceae (Brassica and Arabidopsis Spp.) and Vitales (Vitis vinifera, Citrus sinensis), while it is ineffective with score of excess of 10 among Fabids (Additional file 10: Figure S9A and B). It is important to note that Vitales have not only very high expression of miR403, but also have high AGO2 targeting ability compared to other plant families. We also checked targeting of AGO3 mRNAs by miR403. Such a targeting seem to be possible only among Arabidopsis Spp. (Additional file 11: Figure S10) but not among members of any other plant family. Functional significance of this relationship is not known.

\section{Discussion}

AGO expression levels and susceptibility to viruses

It has been clearly shown that few AGOs in addition to being important for development by controlling transcription factors and other endogenes, are the first line of defense against incoming pathogens. Two lines of evidence are strongly in favor of this observation. Firstly, viral and other pathogen-derived siRNAs are incorporated into AGO1 and to less extent AGO2, thereby performing the role of targeting complementary mRNAs derived from the incoming pathogens themselves. Secondly, pathogens have the ability to selectively target 
these AGOs both at post-transcriptional as well as posttranslational steps. Since AGO1 is responsible for stabilization and activity of most miRNAs, it has been well documented that miRNA levels change generally during pathogenesis, especially during virus infections, due to AGO1 targeting. Unlike most miRNAs that change marginally during viral infections, levels of miR168 changes drastically. This specific change is likely brought about by viral counter-defense proteins, but the mechanism is largely unknown. However, it has been clearly shown that this interaction is crucial for pathogenicity. Thus, absence or lower expression of miR168 in some plants (Figure 6) may indicate a higher expression of AGO1 in those species and this is where viruses might need to target AGO1 as part of their counter defense strategy. In line with this hypothesis, virus-mediated induction of miR168 has been observed among those plants where miR168 levels are generally lower in the absence of viral infections. On the other hand, higher expression of miR168 in some plant lineages might be resulting in a lower accumulation of corresponding AGOs. Indeed in leaves and roots of rice, a monocot, where miR168 levels are quite high, expression of AGO1s is comparatively at lower levels than in Arabidopsis [40,41]. In such cases, viruses need not target AGO1 as part of their counter-defense simply because antiviral role by AGO1 may be negligible or is taken over by other AGOs. This may be the case for rice, where neither a strong induction of miR168, nor a corresponding reduction in AGO1 levels has been observed upon viral infections (Additional file 8: Figure S7, [38]).

On the other hand, absence of miR403 among monocots correlates well with the expression levels of AGO2. In rice, AGO2 expression is very high among all tissues unlike in Arabidopsis where AGO2 expression is largely confined to siliques and at much lower levels in leaves $[40,41]$. It remains to be investigated if AGO2 indeed acts as an antiviral AGO among monocots. However, unlike in Arabidopsis where AGO2 gets induced during viral infections [17], there is hardly any change in AGO2 levels during Rice stripe virus and Rice dwarf virus infections in rice [38]. This supports our view that induction and regulation of AGOs by viruses is restricted to few plant lineages.

\section{Rapid evolution of tobacco miR168 isoforms}

A case for loss of miRNAs can be argued based on the miR168 diversity in $N$. tabacum. The common dicot forms of miR168 in tobacco (isoforms $d$ and e) have insertions of MITE-like transposons. A direct result of this insertion corresponds to reduced accumulation of mature miRNA forms in all tissue types. The Solanaceae-specific forms on the other hand ( $\mathrm{a}, \mathrm{b}$ and $\mathrm{c}$ ), does not have MITE-like insertions and are expressed at high levels. A duplication event of miR168 in tobacco might have ended up with isoforms having two different miR168 sequences. One set ( $\mathrm{d}$ and e) while retaining mature miR168 of dicot ancestor, attracted transposons in the loop region. Tobacco seems to be a hotbed for miR168 duplication since it already has the most diverse miR168 precursors. In addition, there are many tobacco genomic regions matching miR168 sequences present in the genome, sometimes their $\mathrm{miR}^{*}$ sequences either missing or present a long distance downstream or upstream. These may represent the lost miR168 isoforms. It is possible that miR168 example that is observed in tobacco is seen in other miRNAs where some forms are lost during duplication and subsequent loss.

Transposons invite siRNAs and methylation. Invasive Transposons such as MITES can be detected and neutralized using RNA-directed DNA methylation [42]. This may be how promoters of some miRNAs become methylated and inactive. It is possible thus that tobacco miR168 $\mathrm{d}$ and e isoforms may have been targets of RNA-directed DNA methylation and hence no longer express their RNAs. It will be interesting to speculate if viruses can still modulate expression of tobacco miR168d and e. Unfortunately, there are no small RNA datasets from tobacco infected with viruses to see if miR168 d and e isoforms are induced upon virus infection. However, other line of evidence suggests that these isoforms are not likely induced upon virus infections. Usually upon virus infections, not just mature miR168, but also its stem-loop structures over-accumulate [16]. However, miR168d and e may not be induced by viruses as seen from the absence of accumulation of their precursors or pre-miRNAs [16]. A stemloop of miR168 d and e (they are around $200+\mathrm{nts}$ ) was not seen among tobacco samples infected with viruses.

\section{Conclusions}

Our study indicates that intricacies of AGO targeting by miRNAs as observed in Arabidopsis is specific only among few plant lineages. Plants have evolved every shade of this regulation providing case for varying miRNA levels, thus influencing transcription factor and other activities regulated by miRNAs. The nature of this interaction may also influence disease resistance due to the way viruses use these miRNAs to manage the arms race with their host plants.

\section{Methods}

Identifying miRNA Diversity, Sequence Alignments and their Targets

The UEA small RNA analysis toolkit [32] was used to identify members of a given miRNA family (miRProf and miRCat) using default as well as allowing 3 mismatches. Detailed description of the tool is given in http://srnatools.cmp.uea.ac.uk/. Sequences of miR168 and miR403 were obtained from miRBase release 20 [43,44] and aligned using ClustalW and ClustalX2 [45]. Previously 
unreported sequences of miR168 and miR403 were obtained from EST datasets after fulfilling criteria for plant miRNAs including checking for secondary structure (RNAfold, [32]) as well as abundance and distribution of small RNAs across the length of the precursors. List of all plants species and their families are given in Additional file 1: Table S1. Targets of miRNA were identified using two different algorithms, namely, psRNATarget algorithm [25] and TAPIR algorithm [26]. To find targets of miR168 and miR403 family in the plant genomes, target AGO sequences were taken from the NCBI [46], Solanaceous genome Network [47], Refseq [48] as well as from NCBI GEO [49].

Analysis of abundance of miR168 and miR403 members were performed through miRProf analysis of published large-scale data sets derived from various plant species available through the Gene Expression Omnibus (GEO) platform [49,50]. These libraries have been described previously $[5,6,12,30,51-57]$. miRNA sequences were checked to compensate for the mis annotation of miR168 type and miR403-type sequences in miRBase.

\section{Phylogenetic analysis}

The phylogenetic tree was constructed using the MEGA 6.0 software with Neighbor Joining Method with 1000 bootstrap replications. The model used was Jukes Cantor that had the highest log-likelihood score according to the J-model Test (https://code.google.com/p/jmodeltest2/). For the J-model test the precursor alignment was given as the input in ".aln" format. It calculates for the variations in the nucleotide sequences and gives the log-likelihood scores for all the models for phylogenetic tree construction.

\section{Additional files}

Additional file 1: Table S1. Plant species used for small RNA analysis. Additional file 2: Figure S1. Phylogenetic analysis of precursors of miR168.

Additional file 3: Figure S2. Targeting abilities of miR168 among selected plants indicating mosaic targeting among multiple AGO1 members in monocots.

Additional file 4: Figure S3. Length of loops (distance between miRNA and miRNA*) among 58 miR168 precursors from diverse plants.

Additional file 5: Figure S4. Secondary structures of 58 precursors of miR168 indicating clade-specific changes in the loop region.

Additional file 6: Figure S5. Multiple sequence alignment of miR168 precursors from Solanaceae indicating site of MITE insertion.

Additional file 7: Figure S6. Abundance and sequence diversity of miR168 members across plant families in reproductive tissues.

Additional file 8: Figure S7. miR168 is not significantly induced in rice upon infection with viruses.

Additional file 9: Figure S8. Abundance and sequence diversity of miR403 members across plant families in reproductive tissues.

Additional file 10: Figure S9. Ago2 targeting abilities of miR403 across plants.

Additional file 11: Figure S10. Ago3 targeting by miR403.

\section{Competing interests}

The authors declare that they have no competing interests.

\section{Authors' contributions}

PVS conceived and designed the experiments. SJ and PVS carried out the experiments. SJ carried out miRNA sequence analysis. Both authors drafted and revised the manuscript and have read and approved the final version.

\section{Acknowledgements}

PVS acknowledges support from Ramanujan Fellowship (SR/S2/RJN-109/2012; Department of Science and Technology, Government of India). The PI's lab is supported by NCBS-TIFR core funding and funds from Department of Biotechnology, Govt. of India. We thank Rahul Raj Singh and N.D. Sunitha for comments.

Received: 25 August 2014 Accepted: 12 November 2014 Published: 2 December 2014

\section{References}

1. Baulcombe D: RNA silencing in plants. Nature 2004, 431:356-363.

2. Voinnet O: Origin, biogenesis, and activity of plant microRNAs. Cell 2009, 136:669-687.

3. Axtell MJ: Evolution of microRNAs and their targets: are all microRNAs biologically relevant? Biochim Biophys Acta 2008, 1779:725-734.

4. Cuperus JT, Fahlgren N, Carrington JC: Evolution and Functional Diversification of MIRNA Genes. Plant Cell 2011, 23:431-442.

5. Zhai J, Jeong D-H, De Paoli E, Park S, Rosen BD, Li Y, Gonzalez AJ, Yan Z, Kitto SL, Grusak MA, Jackson SA, Stacey G, Cook DR, Green PJ, Sherrier DJ, Meyers BC: MicroRNAs as master regulators of the plant NB-LRR defense gene family via the production of phased, trans-acting siRNAs. Genes Dev 2011, 25:2540-2553.

6. Shivaprasad PV, Chen H-M, Patel K, Bond DM, Santos BACM, Baulcombe DC: A microRNA superfamily regulates nucleotide binding site-leucine-rich repeats and other mRNAs. Plant Cell 2012, 24:859-74.

7. Li F, Pignatta D, Bendix C, Brunkard JO, Cohn MM, Tung J, Sun H, Kumar P, Baker B: MicroRNA regulation of plant innate immune receptors. Proc Natl Acad Sci 2012, 109:1790-1795.

8. Taylor RS, Tarver JE, Hiscock SJ, Donoghue PCJ: Evolutionary history of plant microRNAs. Trends Plant Sci 2014, 19:175-182.

9. Willmann MR, Poethig RS: Conservation and evolution of miRNA regulatory programs in plant development. Curr Opin Plant Biol 2007, 10:503-511.

10. Jones-Rhoades MW: Conservation and divergence in plant microRNAs. Plant Mol Biol 2012, 80:3-16.

11. Felippes FF, Schneeberger K, Dezulian T, Huson DH, Weigel D: Evolution of Arabidopsis thaliana microRNAs from random sequences. RNA 2008, 14:2455-2459.

12. Montes RAC, de Fátima Rosas-Cárdenas F, De Paoli E, Accerbi M, Rymarquis LA, Mahalingam G, Marsch-Martínez N, Meyers BC, Green PJ, de Folter S: Sample sequencing of vascular plants demonstrates widespread conservation and divergence of microRNAs. Nat Commun 2014, 5:3722.

13. Xie Z, Kasschau KD, Carrington JC: Negative feedback regulation of Dicer-Like1 in Arabidopsis by microRNA-guided mRNA degradation. Curr Biol 2003, 13:784-789.

14. Vaucheret H: Plant ARGONAUTES. Trends Plant Sci 2008, 13:350-358.

15. Vaucheret H, Vazquez F, Crete P, Bartel DP: The action of ARGONAUTE1 in the miRNA pathway and its regulation by the miRNA pathway are crucial for plant development. Genes Dev 2004, 18:1187-1197.

16. Várallyay E, Válóczi A, Agyi A, Burgyán J, Havelda Z: Plant virus-mediated induction of miR168 is associated with repression of ARGONAUTE1 accumulation. EMBO J 2010, 29:3507-3519.

17. Harvey JJW, Lewsey MG, Patel K, Westwood J, Heimstadt S, Carr JP, Baulcombe DC: An Antiviral Defense Role of AGO2 in Plants. PLOS One 2011, 6 .

18. McMorran JP, Allen TC: Maintenance, symptoms and distribution of potato viruses $\mathrm{X}, \mathrm{S}, \mathrm{Y}, \mathrm{A}, \mathrm{M}$ and leafroll in potato tissue culture plantlets. Am PotatoJ 1983, 60:839-848.

19. Allen E, Xie Z, Gustafson AM, Carrington JC: microRNA-Directed Phasing during Trans-Acting siRNA Biogenesis in Plants. Cell 2005, 121:207-221.

20. Rhoades MW, Reinhart BJ, Lim LP, Burge CB, Bartel B, Bartel DP: Prediction of plant microRNA targets. Cell 2002, 110:513-520. 
21. Mallory AC, Vaucheret $\mathrm{H}$ : Functions of micro-RNAs and related small RNAs in plants. Nat Genet Supp/ 2006, 38:S31.

22. Vaucheret $\mathrm{H}$, Mallory AC, Bartel DP: AGO1 Homeostasis entails coexpression of MIR168 and AGO1 and preferential stabilization of miR168 by AGO1. Mol Cell 2006, 22:129-136.

23. Allen E, Xie Z, Gustafson AM, Sung G-H, Spatafora JW, Carrington JC: Evolution of microRNA genes by inverted duplication of target gene sequences in Arabidopsis thaliana. Nat Genet 2004, 36:1282-1290.

24. Vazquez F, Blevins T, Ailhas J, Boller T, Meins F: Evolution of Arabidopsis MIR genes generates novel microRNA classes. Nucleic Acids Res 2008, 36:6429-6438

25. Dai XB, Zhao PX: psRNATarget: a plant small RNA target analysis server. Nucleic Acids Res 2011, 39:W155-W159.

26. Bonnet $\mathrm{E}$, He $\mathrm{Y}$, Billiau K, Van de Peer $\mathrm{Y}$ : TAPIR, a web server for the prediction of plant microRNA targets, including target mimics. Bioinformatics 2010, 26:1566-1568.

27. Srivastava PK, Moturu TR, Pandey P, Baldwin IT, Pandey SP: Comparison of performance of plant miRNA target prediction tools and the characterization of features for genome-wide target prediction. BMC G 2014, 15:348.

28. Tang $\mathrm{H}$, Bowers JE, Wang $\mathrm{X}$, Paterson AH: Angiosperm genome comparisons reveal early polyploidy in the monocot lineage. Proc Natl Acad Sci U S A 2010, 107:472-477.

29. Colaiacovo M, Lamontanara A, Bernardo L, Alberici R, Crosatti C, Giusti L, Cattivelli $L$, Faccioli P: On the complexity of miRNA-mediated regulation in plants: novel insights into the genomic organization of plant miRNAs. Biology Direct 2007, 7:15.

30. Song $L$, Axtell MJ, Fedoroff NV: RNA Secondary Structural Determinants of miRNA Precursor Processing in Arabidopsis. Curr Biol 2010, 20:37-41.

31. Piriyapongsa J, Mariño-Ramírez $L$, Jordan IK: Origin and evolution of human microRNAs from transposable elements. Genetics 2007, 176:1323-1337.

32. Moxon S, Schwach F, Dalmay T, MacLean D, Studholme DJ, Moulton V: A toolkit for analysing large-scale plant small RNA datasets. Bioinformatics 2008, 24:2252-2253.

33. Baumberger N, Tsai C-H, Lie M, Havecker E, Ziegler-Graff V, Baulcombe DC: The Polerovirus Silencing Suppressor PO Targets ARGONAUTE Proteins for Degradation. Curr Biol 2007, 17:1609-1614.

34. Moissiard G, Voinnet O: Viral suppression of RNA silencing in plants. Mol Plant Pathol 2004, 5:71-82.

35. Chapman EJ, Prokhnevsky Al, Gopinath K, Dolja V, Carrington JC: Viral RNA silencing suppressors inhibit the microRNA pathway at an intermediate step. Genes Dev 2004, 18:1179-1186.

36. Azevedo J, Garcia D, Pontier D, Ohnesorge S, Yu A, Garcia S, Braun L, Bergdoll M, Hakimi MA, Lagrange T, Voinnet O: Argonaute quenching and global changes in Dicer homeostasis caused by a pathogen-encoded GW repeat protein. Genes Dev 2010, 24:904-915.

37. Du P, Wu J, Zhang J, Zhao S, Zheng H, Gao G, Wei L, Li Y: Viral infection induces expression of novel phased microRNAs from conserved cellular microRNA precursors. PLoS Pathog 2011, 7:e1002176

38. Satoh K, Shimizu T, Kondoh H, Hiraguri A, Sasaya T, Choi IR, Omura T, Kikuchi S: Relationship between symptoms and gene expression induced by the infection of three strains of Rice dwarf virus. PLoS One 2011, 6:e18094.

39. Sunkar $R$, Jagadeeswaran $G$ : In silico identification of conserved microRNAs in large number of diverse plant species. BMC Plant Biol 2008, 8:37.

40. Yang Y, Zhong J, YDan O, Yao J: The integrative expression and co-expression analysis of the AGO gene family in rice. Gene 2013, 528:221-235.

41. Kapoor M, Arora R, Lama T, Nijhawan A, Khurana JP, Tyagi AK, Kapoor S: Genome-wide identification, organization and phylogenetic analysis of Dicer-like, Argonaute and RNA-dependent RNA Polymerase gene families and their expression analysis during reproductive development and stress in rice. BMC Genomics 2008, 9:451.

42. Marí-Ordóñez A, Marchais A, Etcheverry M, Martin A, Colot V, Voinnet O: Reconstructing de novo silencing of an active plant retrotransposon. Nat Genet 2013, 45:1029-39.

43. Kozomara A, Griffiths-Jones S: miRBase: integrating microRNA annotation and deep-sequencing data. Nucleic Acids Res 2011, 39:D152-D157.
44. Griffiths-Jones S, Grocock RJ, van Dongen S, Bateman A, Enright AJ: miRBase: microRNA sequences, targets and gene nomenclature. Nucleic Acids Res 2006, 34:D140-D144.

45. Larkin MA, Blackshields G, Brown NP, Chenna R, McGettigan PA, McWilliam $H$, Valentin F, Wallace IM, Wilm A, Lopez R, Thompson JD, Gibson TJ, Higgins DG: Clustal W and clustal X version 2.0. Bioinformatics 2007, 23:2947-2948

46. Johnson M, Zaretskaya I, Raytselis Y, Merezhuk Y, McGinnis S, Madden TL: NCBI BLAST: a better web interface. Nucleic Acids Res 2008, 36:W5-9.

47. Mueller LA, Mills AA, Skwarecki B, Buels RM, Menda N, Tanksley SD: The SGN comparative map viewer. Bioinformatics 2008, 24:422-423.

48. Pruitt KD, Tatusova T, Maglott DR: NCBI reference sequences (RefSeq): A curated non-redundant sequence database of genomes, transcripts and proteins. Nucleic Acids Res 2007, 35:D61-65.

49. Barrett T, Troup DB, Wilhite SE, Ledoux P, Rudnev D, Evangelista C, Kim IF, Soboleva A, Tomashevsky M, Edgar R: NCBI GEO: Mining tens of millions of expression profiles - Database and tools update. Nucleic Acids Res 2007, 35:D76-765.

50. Edgar R, Domrachev M, Lash AE: Gene Expression Omnibus: NCBI gene expression and hybridization array data repository. Nucleic Acids Res 2002, 30:207-210.

51. Pilcher RLR, Moxon S, Pakseresht N, Moulton V, Manning K, Seymour G, Dalmay T: Identification of novel small RNAs in tomato (Solanum lycopersicum). Planta 2007. doi:10.100.

52. Havecker ER, Wallbridge LM, Hardcastle TJ, Bush MS, Kelly KA, Dunn RM, Schwach F, Doonan JH, Baulcombe DC: The Arabidopsis RNA-Directed DNA Methylation Argonautes Functionally Diverge Based on Their Expression and Interaction with Target Loci. Plant Cell 2010, 22:321-334.

53. Ma ZR, Coruh C, Axtell MJ: Arabidopsis lyrata Small RNAs: Transient MIRNA and Small Interfering RNA Loci within the Arabidopsis Genus. Plant Cell 2010, 22:1090-1103.

54. Molnar A, Melnyk CW, Bassett A, Hardcastle TJ, Dunn R, Baulcombe DC: Small silencing RNAs in plants are mobile and direct epigenetic modification in recipient cells. Science 2010, 328:872-875.

55. Wang C, Wang XC, Kibet NK, Song CN, Zhang CQ, Li XY, Han J, Fang JG: Deep sequencing of grapevine flower and berry short RNA library for discovery of novel microRNAs and validation of precise sequences of grapevine microRNAs deposited in miRBase. Physiol Plant 2011, 143:64-81.

56. Shivaprasad PV, Dunn RM, Santos BA, Bassett A, Baulcombe DC: Extraordinary transgressive phenotypes of hybrid tomato are influenced by epigenetics and small silencing RNAs. EMBO J 2012, 31:257-266.

57. Song X, Li P, Zhai J, Zhou M, Ma L, Liu B, Jeong DH, Nakano M, Cao S, Liu C, Chu C, Wang XJ, Green PJ, Meyers BC, Cao X: Roles of DCL4 and DCL3b in rice phased small RNA biogenesis. Plant J 2011, 69:462-474.

\section{doi:10.1186/1471-2164-15-1049}

Cite this article as: Jagtap and Shivaprasad: Diversity, expression and mRNA targeting abilities of Argonaute-targeting miRNAs among selected vascular plants. BMC Genomics 2014 15:1049

\section{Submit your next manuscript to BioMed Central and take full advantage of:}

- Convenient online submission

- Thorough peer review

- No space constraints or color figure charges

- Immediate publication on acceptance

- Inclusion in PubMed, CAS, Scopus and Google Scholar

- Research which is freely available for redistribution 